\title{
Research
}

\section{Worrying about wasting GP time as a barrier to help-seeking:}

\author{
a community-based, qualitative study
}

\begin{abstract}
Background

Worrying about wasting GP time is frequently cited as a barrier to help-seeking for cancer symptoms.
\end{abstract}

Aim

To explore the circumstances under which individuals feel that they are wasting GP time.

\section{Design and setting}

Community-based, qualitative interview studies that took place in London, the South East and the North West of England.

\section{Method}

Interviewees $(n=62)$ were recruited from a sample ( $n=2042$ ) of adults aged $\geq 50$ years who completed a 'health survey' that included a list of cancer 'alarm' symptoms. Individuals who reported symptoms at baseline that were still present at the 3-month follow-up ( $n=271)$, and who had also consented to be contacted ( $n=215$ ), constituted the pool of people invited for interview. Analyses focused on accounts of worrying about wasting GP time.

\section{Results}

Participants were worried about wasting GP time when time constraints were visible, while dismissive interactions with their GP induced a worry of unnecessary help-seeking. Many felt that symptoms that were not persistent, worsening, or life-threatening did not warrant GP attention. Additionally, patients considered it time-wasting when they perceived attention from nurses or pharmacists to be sufficient, or when appointment structures (for example, 'one issue per visit') were not adhered to. Close relationships with GPs eased worries about time-wasting, while some patients saw GPs as fulfilling a service financed by taxpayers.

\section{Conclusion}

Worrying about wasting GP time is a complex barrier to help-seeking. GP time and resource scarcity, symptom gravity, appointment etiquette, and previous GP interactions contribute to increasing worries. Friendly GP relationships, economic reasoning, and a focus on the GP's responsibilities as a medical professional reduce this worry.

\section{Keywords}

cancer; diagnosis; help-seeking behaviour neoplasms; primary health care.

\section{INTRODUCTION}

International comparisons show that 1-year cancer survival rates are worse in the UK compared with countries with similar healthcare systems. ${ }^{1,2}$ Previous patient experience, ${ }^{3}$ and the perception of 'warranting' medical attention, can lead to delays in help-seeking. ${ }^{4}$ Warranting medical attention may be influenced by several factors, including the type of symptoms, ${ }^{5}$ awareness of the significance of the symptoms, ${ }^{6,7}$ perception of personal risk of cancer, ${ }^{8}$ and psychological, social, and physical barriers to health care. ${ }^{9}$

In several qualitative investigations, patients mentioned the worry of wasting their GP's time' as a psychological barrier to help-seeking for symptoms indicative of various cancers. ${ }^{10,11}$ In a recent study, $15 \%$ of responders stated that worry about wasting their doctor's time was an anticipated barrier to healthcare seeking. ${ }^{12}$ A qualitative synthesis showed that fear of being labelled a time-waster was a barrier to seeking help for possible cancer symptoms, ${ }^{13}$ which was also associated with longer anticipated helpseeking intervals. ${ }^{14}$ In some cases, receiving a benign diagnosis after symptomatic presentation can reinforce the fear of being a time-waster. ${ }^{3}$

The worry of wasting GP time reflects the discourse surrounding GP time scarcity and pressures. This perception of timeconstrained doctors is enhanced by long waiting times and an apparent shortage of

SK Cromme, MSc, research assistant;

K Winstanley, PhD, research associate

C Renzi, MD senior clinical research associate;

C Friedemann Smith, DPhil, research associate

J Wardle, PhD, director, Health Behaviour

Research Centre, Epidemiology and Public Health, University College London, London. KL Whitaker,

$\mathrm{PhD}$, senior lecturer, School of Health Sciences,

Faculty of Health and Medical Sciences, University of Surrey, Guildford.

Address for correspondence

Katriina L Whitaker, School of Health Sciences, appointments, ${ }^{15}$ which may lead patients to present symptoms at a later stage, once they are confident that they are eligible for medical attention. Patients who seek help quickly have few reservations about using doctors' time, and believe that doctors could offer reassurance, ${ }^{15}$ symptom alleviation, or a referral to specialist care. ${ }^{13}$

Informing patients of the costs of their NHS appointments or requesting a fee for visiting their GP are some of the political discussions portrayed by the media. ${ }^{16}$ Importantly, not understanding the underlying mechanisms that feed into the worry of wasting a GP's time may lead to adverse effects of such media portrayal. For example, instead of increasing awareness of GP time scarcity in individuals who go to the GP unnecessarily, it may increase worry about GP time in those who are already concerned.

Although 'wasting GP time' is often referred to as a potent factor leading to delay in symptom presentation, this psychological barrier has not been explored in depth with people in the community experiencing cancer alarm symptoms. The aim of the present study was to examine the different meanings given to the concept of wasting GP time, by deconstructing the decision-making process that leads individuals to feel that they are wasting, or are not wasting, their GP's time.

\section{METHOD}

A detailed description of the study methods, including a recruitment flow chart, has been

Faculty of Health and Medical Sciences, Duke of Kent Building, University of Surrey, Guildford, Surrey, GU2 7XH, UK.

E-mail: k.whitakerdsurrey.ac.uk

Submitted: 1 February 2016; Editor's response: 1 March 2016; final acceptance: 15 March 2016. (CBritish Journal of General Practice

This is the full-length article (published online 24 May 2016) of an abridged version published in print. Cite this version as: Br J Gen Pract 2016; DOI: 10.3399/bjgp16X685621 


\section{How this fits in}

Worrying about wasting GP time is a commonly cited barrier to medical helpseeking in population-based surveys, and contributes to longer time-to-symptom presentation. However, there has been very little qualitative work on the concept of wasting GP time. One qualitative study with people reporting cancer 'alarm' symptoms highlighted the perceived importance of limiting demands on GPs, but did not explore the concept in detail. This is the first qualitative study to explore what people experiencing cancer symptoms mean by 'wasting their GP's time'. The findings highlight potential avenues to mitigate against this help-seeking barrier and encourage prompt symptomatic presentation.

published previously; ${ }^{15}$ a brief summary is provided here.

\section{Participant selection and recruitment}

Participants were recruited from responders $(n=2042)$ to a large prospective postal survey conducted through four GP practices in England, which had been posted to 4913 individuals aged $\geq 50$ years in October 2013. Responders reporting at least one cancer alarm symptom ( $n=936 / 2042 ; 46 \%$ ), and who had agreed to be contacted again ( $n=602 / 936$; 64\%), were sent a follow-up survey after 3 months to capture symptom experiences over time; $75 \%$ of people (450/602) returned the survey. Inclusion criteria for the in-depth interviews were: experience of at least one of 14 'alarm' symptoms at the 3-month follow-up (271/450; 60\%), and consent to be contacted for interview (215/271; 79\%). Of the 215 responders meeting the inclusion criteria, 144 were consequently invited to interview, with a response rate of $60 \%$ (86/144). Invitees were purposively sampled based on sex, age, and geographic area of residence. $^{3}$ Interviews ceased once data saturation was achieved $(n=62)$.

\section{Interviews}

Interview data were collected from people reporting ongoing cancer 'alarm' symptoms. After the initial narrative account, a semi-structured topic guide was used to ensure topics such as help-seeking and psychological barriers (wasting GP timel were explored. Prompts were used to initiate responses to the 'wasting GP time' component if it did not arise spontaneously. Participants were asked to tell whether they had had similar experiences to other interviewees; for example, Some people mentioned that they avoid or delay seeing the GP because they worry about wasting the doctor's time or bothering the doctor. Does this ever apply to you?' Interviews lasted on average 42 minutes (range: 22-66 minutes).

\section{Analysis}

Demographic and basic frequency information was analysed using SPSS version 21.0. Interviews were digitally recorded and transcribed verbatim. Two of the authors read and reread the transcripts and allocated arising themes to the two following questions: "When do people feel as though they are wasting GP time?' and What are the accounts of people who use GP services freely?' Transcripts were coded using NVivo 9.0 software, and the emerging themes were managed in Microsoft Excel. Emerging sub-themes were discussed in frequent meetings, and agreed by the co-authors using an iterative process.

\section{RESULTS}

The demographic characteristics of the sample can be seen in Table 1. The type of symptoms and help-seeking reported in the interview sample have been reported previously. ${ }^{15}$ The most common symptoms were persistent cough/hoarseness (27\%) and persistent change in bladder habits (24\%). The least common symptoms were unexplained bleeding and blood in urine (both 3\%). Table 2 illustrates six subheadings and 11 themes that arose under the two questions, supported by participants' quotations.

\section{When do people feel like they are wasting GP time?}

When time constraints are visible. When discussing their reservations about seeking help for cancer 'alarm' symptoms, participants made frequent reference to the time constraints incurred by GPs. Reference was made to increasing duties on GPs alongside their medical responsibilities such as '... all the ... paperwork they have got to do and the money juggling that the government is forcing on them: (P18, M, 50 years; change in bowel habits, rectal bleeding)

Participants were understanding of GPs and how '... they are not going to have time to do this, that, and the other. It's the system.' (P29, F, 83 years; changes in the appearance of a mole), and mentioned how this made them '... reluctant to be knocking on the GP's door all the time because we 
Table 1. Demographic characteristics of participants $(n=62)$

\begin{tabular}{|c|c|}
\hline Variable & $n(\%)$ \\
\hline \multicolumn{2}{|l|}{ Sex } \\
\hline Male & 33 (53.2) \\
\hline Female & $29(46.8)$ \\
\hline \multicolumn{2}{|l|}{ Age, years } \\
\hline Mean (range) & $65(50.3-92.7)$ \\
\hline \multicolumn{2}{|l|}{ Ethnicity } \\
\hline White/white British & 57 (91.9) \\
\hline Other & $5(8.1)$ \\
\hline \multicolumn{2}{|l|}{ Educational level $^{a}$} \\
\hline University degree & $28(45.2)$ \\
\hline Higher qualification below degree & $13(21)$ \\
\hline Lower qualification & $21(33.9)$ \\
\hline
\end{tabular}

know how busy they are.' (P21, F, 67 years; change in bowel habits, abdominal bloating, change in the appearance of a mole).

The perception of GPs' lack of time was derived in particular from waiting times at the practice and difficulty making an appointment: 'They have got a hard enough job as it is. As / say, you've got to wait a week for an appointment anyway.' (P22, M, 68 years; persistent cough or hoarseness)

Furthermore, negative interactions where participants were left with the impression that they were 'crying wolf too much' (P11, M, 69 years; change in bowel habits) made participants feel as though they were wasting their GP's time. At times, this made help-seeking humiliating and made some feel as though GPs 'are just not interested' (P54, F, age missing; persistent unexplained pain): Well, it was just so overwhelmingly humiliating, the fact that I went in and said, "Please can you do something with this?" And he literally turned round and said, "Why are you bothering me with something as trivial as that?"' (P24, F, 64 years; persistent cough or hoarseness]

Although negative experiences with GPs led some to feel bad about help-seeking, others reported how positive GP interactions reduced their worries about wasting time, pointing out that the GP 'doesn't make you feel that you're a nuisance. '(P20, M, 67 years; persistent cough or hoarseness)

Some conveyed a need to avoid being perceived as a hypochondriac when returning with a symptom. They expressed how they did not want to bother the doctor: ' do feel as though I am ... not annoying them, but "oh not you again", sort of thing.' (P62,
M, 62 years; persistent unexplained pain, abdominal bloatingl

In some cases, such contemplation occurred despite acknowledging that they did not know how the doctor felt or claiming that they were not scared of what he [GP] might think of me, exactly, but I don't want to bother him.' (P36, F, 72 years; sore that does not heal, persistent unexplained pain)

Participants mentioned that: 'It's quite important to respect the fact they have these very limited appointments now. So I try not to take advantage of that and mention anything else.' (P42, F, age missing; change in bowel habits, persistent cough or hoarseness)

Thus, to avoid wasting GP time, participants not only held back information so that they would not compromise their GP's appointment structure, but some also felt they had to be apologetic if they did not stick to the 'one item per visit' rule or the 10-minute time slot. One participant said he would: rehearse what you are going to tell the doctor, because I'm very, very conscious of they've only got so much time for each patient.' (P53, M, 58 years; unexplained lump)

When symptoms are perceived as not serious enough. Some participants felt that seeking help for symptoms that were not serious enough would result in wasting GP time. Reference was made to symptom characteristics, such as whether they were life-threatening, treatable, or chronic. Some said they '... wouldn't want to go and see them [GP] with something which was trivial' (P5, M, 62 years; sore that does not heal) or 'It's got to be life-threatening, and that requires investigations.' (P22, M, 68 years; persistent cough or hoarseness)

Some described withholding information about less serious symptoms, believing they would otherwise waste their GP's time. For example, they made reference to not wanting to: '... bother the GP with all my symptoms every time, just what I think is pertinent: (P19, F, 67 years; unexplained weight loss, persistent cough or hoarseness)

Where long waiting times existed, there appeared to be a link between awareness of GP time constraints, symptom seriousness, and the worry of wasting GP time, and potentially benign symptoms increased worry over wasting GP time. Some participants felt that: '... the longer the waiting list, the more urgent I think it needs to be in order to go.' (P9, M, 63 years; change in bladder habits, sore that does not heal)

Many participants only felt comfortable seeking help for symptoms that were 


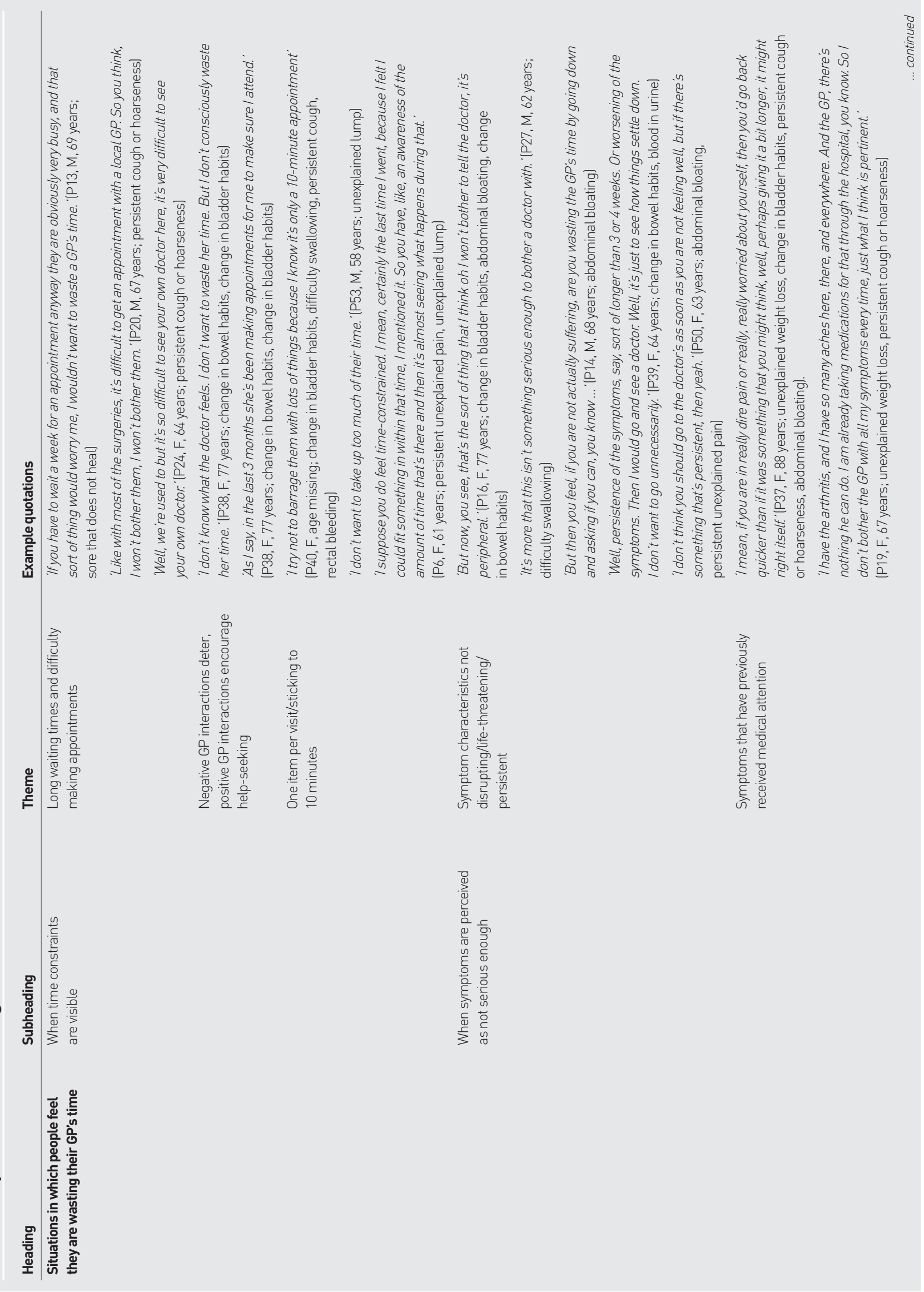




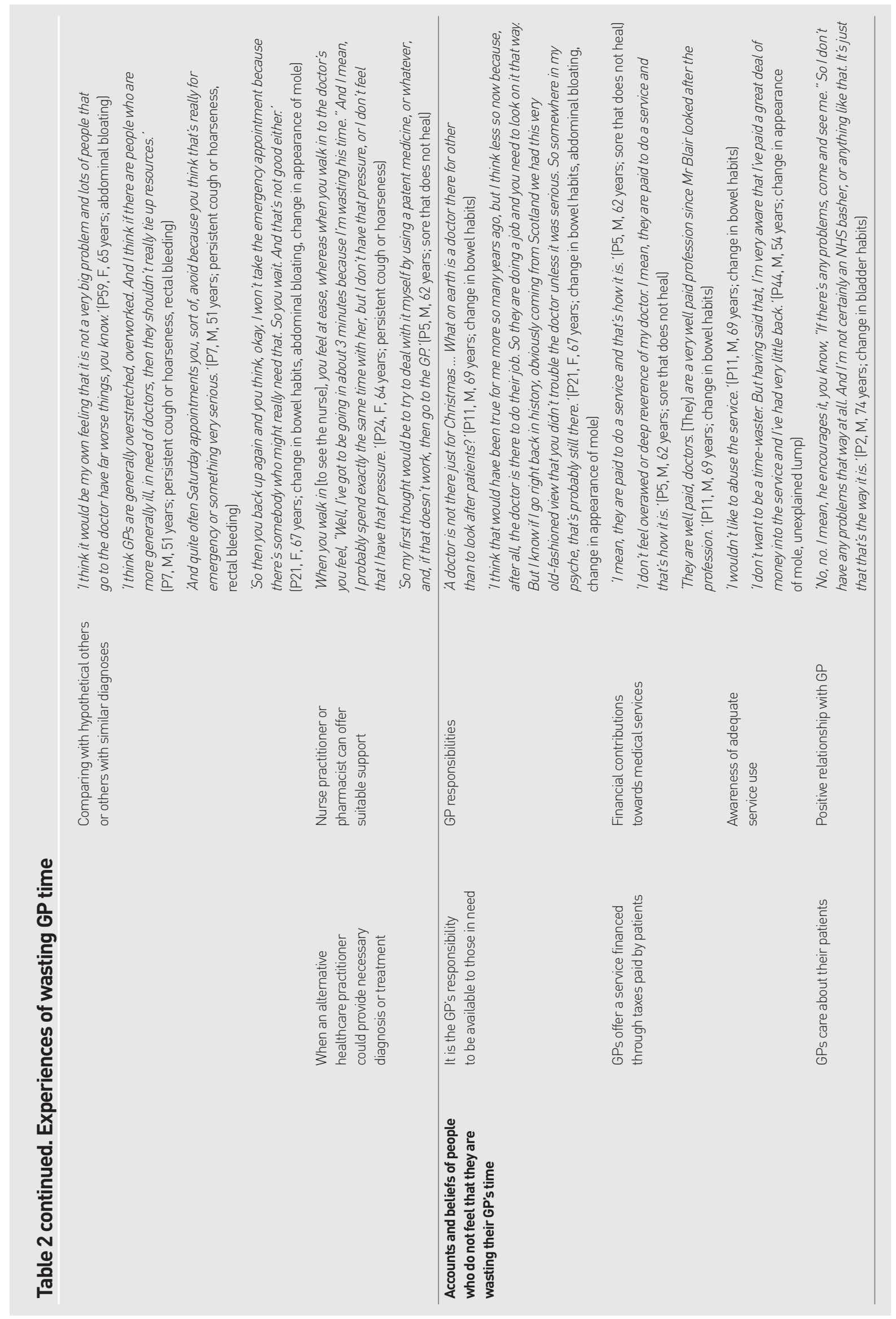


'worsening' or 'persistent', as these characteristics were seen to reduce the likelihood of going unnecessarily. Although 'persistent', symptoms could also be problematic because people do not want to go back unless you are in really dire pain. (P37, F, 88 years; unexplained weight loss, change in bladder habits, persistent cough or hoarseness, abdominal bloatingl

Symptom characteristics also contributed to how individuals compared their symptoms or situation to hypothetical others who may be in more urgent need of medical attention. Taking hypothetical others into account increased their worry of wasting their GP's time. Some felt uncomfortable ... bothering the doctor, they have got people who are much more seriously ill to see to than I.' (P10, M, 77 years; abdominal bloating, persistent cough or hoarseness), and that '... other people have more serious things wrong. My things are just trivial.' (P17, M, 62 years, change in bladder habits)

When an alternative healthcare practitioner could provide a diagnosis or treatment. Participants felt that sometimes an alternative healthcare practitioner, such as a nurse, a pharmacist, or even self-medication, could provide a diagnosis or treatment, and that GP time was wasted were these alternative sources of medical attention not sought before a visit to the GP. Going to the pharmacy or self-medicating was a way for participants to 'cope with it elsewhere' (P27, M, 62 years; difficulty swallowingl and to take care of this myself.' (P19, F, 67 years; unexplained weight loss, persistent cough or hoarseness)

Nurse practitioners were seen as quite useful for going along with something that you just want reassurance about.' (P40, $\mathrm{F}$, age missing; change in bladder habits, difficulty swallowing, persistent cough or hoarseness, rectal bleedingl

Reference was made to how participants felt pressured during GP appointments compared with feeling at ease with a nurse. Participants would categorise their symptoms and reasons for a healthcare visit. If symptoms were not serious, and if the outcome sought was reassurance, then visiting a nurse or pharmacist was considered appropriate.

\section{What are the accounts from participants who use GP services freely?}

It is the GP's responsibility to be available to those in need. Those who used GP services freely focused on the GP's responsibilities and obligations as a healthcare provider, but also as an employee of the government through the NHS. These individuals felt that the GP's responsibility is primarily to: ... help us when we [are in] need. So it's not a question of bothering the doctor. (P19, F, 67 years; unexplained weight loss, persistent cough or hoarseness)

Some participants argued that ill people secured the GP's job and that seeking help is therefore ... not wasting his time; he is there to do this job. After all, if it was not for us sick people there, what would the GP do?' (P2, M, 74 years; change in bladder habits)

This rationalisation of the GP's role strengthened their attitude: If I want to go, / go.' (P38, F, 77 years; change in bowel habits, change in bladder habits)

GPS offer a service financed through taxes paid by patients. A further justification for using GP services freely was the observation that GPs offer a service financed by taxpayers. Participants felt entitled to go to the GP' because 'I pay my taxes. I've got a pension and I pay a hefty $20 \%$ of that.' (P58, M, 74 years; change in bladder habits, change in appearance of mole), and because I'm wanting some sort of reassurance and I'm paying for it.' (P30, F. 92 years; persistent cough or hoarseness, change in appearance of mole)

The worry of wasting GP time became secondary to those who felt that medical attention was justified through them having paid towards the NHS in taxes or towards private insurance.

GPS care about their patients. Those who used GP services freely reported having a friendship-like relationship with their GP: ' go because I made friends with my doctor. (P3, $M$, age missing; persistent unexplained pain) They also described confidence in their GP. Those who sought help freely believed that GPs care about their patients: He encourages it (help-seeking), you know, "If there's any problems, come and see me"' (P2, M, 74 years; change in bladder habits)

Some reported that their GP made appointments for them to ensure that they returned regularly: And she [GP] makes the appointment, I don't make the appointment.' (P38, F, 77 years; change in bowel habits, change in bladder habits)

\section{DISCUSSION}

\section{Summary}

As far as the authors are aware, this is the first study to carry out an in-depth investigation about what people experiencing persistent cancer alarm symptoms mean by 'wasting their GP's time' 
and explore situations in which participants felt that they were wasting their GP's time. Understanding how the worry of wasting GP time influences the appraisal process and help-seeking decisions is crucial to improving early symptomatic presentation.

Symptom characteristics strongly influenced whether participants felt they would waste their GP's time or not. However, awareness of time and resource constraints by the GP and the NHS also influenced patients' perception of wasting their GP's time. An awareness of long waiting times and difficulty making appointments led participants to report an increased worry over wasting their GP's time. Participants felt that the longer the waiting times for appointments, the more serious the symptom(s) needed to be. This reasoning offers novel insight into how various components that contribute to the worry of wasting GP time interact with each other. Resource constraints also led to a heightened awareness of what the GP may think of patients for seeking help for particular symptoms. Some participants had concrete ideas of what the GP may think or feel about them despite acknowledging that they did not actually know.

Importantly, this study also investigated the accounts of those who use GP services freely. These individuals felt that due to the taxes they had paid, they were entitled to use the service with moderation and had a general belief that the GP's responsibility was to help those in need and that GPs generally cared about their patients. They also recounted how a good relationship with their GP reduced their worry of wasting their GP's time.

\section{Strengths and limitations}

This study builds on a growing body of research investigating influences on the patient interval (time from noticing a bodily change to seeking medical help), ${ }^{17,18}$ to support individuals to seek medical advice as soon as possible - a key element of the UK government's strategy for cancer, ${ }^{19}$ and the Independent Cancer Taskforce report. ${ }^{20}$ This study offers novel and more detailed insights into the complexities underlying wasting GP time' as a barrier to help-seeking. By interviewing people with ongoing cancer symptoms, but without mentioning cancer, the possible bias introduced in retrospective studies with cancer patients was reduced. ${ }^{11}$ The prospective nature of the present study also meant participants had experienced symptoms for at least 3 months before they were interviewed, reducing the possibility that symptoms were short-lived or transient.
The sample was drawn from London, and South East and North West England, which limits generalisability to other regions. It was also beyond the study's scope to examine the effect of specific symptoms on patients' worry of wasting their GP's time. Investigating patients with more homogeneous symptom groups represents an area for future research; it could provide a more detailed picture and offer a powerful focus for intervention. Another outstanding question is how to balance minimising worry about wasting GP time in those who perceive it as a barrier, rather than readily encouraging all people to contact their GP more often for advice. The study also focused on older men and women laged $\geq 50$ years) because of their higher risk of developing cancer. ${ }^{21}$ However, certain demographic subgroups may be more likely to report worry about wasting their doctor's time, which could be a consideration for future research. For example, in a Danish study, a younger age group (30-49-year-olds) were more likely to report worry about wasting their doctor's time than older age groups. ${ }^{12}$ Finally, GPs were not interviewed and patient-GP interactions were not observed; thus, the GP perspective is notably absent in this account. Future research could interview patient-GP dyads to further explore how interactions may influence help-seeking.

\section{Comparison with existing literature}

Wasting GP time is a barrier to helpseeking frequently mentioned by individuals experiencing cancer 'alarm' symptoms. 10,11,14 As observed in the existing research, participants in the present study felt that, until the symptoms display a certain gravity or until they could categorise them as lifethreatening, help-seeking would be seen by themselves and others as wasting their GP's time." Participants sought to protect their self-identity from being labelled a hypochondriac or 'time-waster' by avoiding help-seeking for symptoms that could be benign, and for symptoms following an 'allclear' diagnosis, ${ }^{3}$ using health care only when they felt it was absolutely necessary. These findings support previous research. 3,13,22

Previous research also showed how physician interaction can influence helpseeking behaviour, by legitimising helpseeking. ${ }^{23}$ In the present study, where GPs came across as abrasive and uninterested, worrying about wasting GP time increased, whereas friendly encounters reduced concern over wasting GP time. Positive interactions with GPs were described in the present qualitative study, in contrast 


\section{Funding}

These analyses were carried out with funding from the Department of Health Policy Research Unit in Cancer Awareness, Screening, and Early Diagnosis, which receives funding for a research programme from the Department of Health Policy Research Programme.

It is a collaboration between researchers from seven institutions (Queen Mary University of London, University College London, King's College London, London School of Hygiene \& Tropical Medicine, Hull York Medical School, Durham University, and Peninsula Medical School). The views expressed are those of the authors and not necessarily those of the NHS or the Department of Health. Katriina L Whitaker was supported by a Cancer Research UK Postdoctoral Fellowship (C33872/A13216). Cristina Renzi was supported by a Cancer Research UK Research Bursary (C48748) A16867).

\section{Ethical approval}

The study materials and protocol were approved by the NHS London Bridge Research Ethics Committee (Reference: 11/LO/1970).

\section{Provenance}

Freely submitted; externally peer reviewed.

\section{Competing interests}

The authors have declared no competing interests.

\section{Open access}

This article is Open Access: CC BY 3.0 license /http://creativecommons.org/ licenses/by/3.0/).

\section{Acknowledgements}

In memory of Professor Jane Wardle (1950-2015).

\section{Discuss this article}

Contribute and read comments about this article: bjgp.org/letters

to previous research where GPs were only mentioned as barriers to help-seeking. ${ }^{11}$

A novel finding was related to the worry of taking GP time away from hypothetical others who may be in more acute need of medical attention. This was a dominant theme during the interviews and highlighted how, when participants compared their own symptoms with those of hypothetical others who were 'much more seriously ill', the worry of wasting GP time increased.

In line with previous research, participants felt they should seek advice from alternative healthcare providers before consulting their GP, ${ }^{11}$ although in the present study this was in the context of not wanting to waste their GP's time, rather than lack of confidence in their GP.

\section{Implications for research and practice}

The appraisal processes of what behaviour constitutes wasting GP time is made up of sociocultural and experience-driven factors that vary from person to person. For practising clinicians, this study highlights areas where they might encourage appropriate help-seeking (for example, providing a safeguard for people reporting vague, non-specific symptoms by making follow-up appointments on behalf of patients). Future studies that capture the discourse of seeking help with GPs and alternative healthcare providers, such as nurse practitioners, in a more in-depth way could offer valuable insight into this promising target for intervention. Focusing on nurse practitioners as a point of contact for symptoms that may not fulfil the subjective requirements for GP attention may not only reduce the barrier represented by 'worrying about wasting GP time', but may also help to distribute demands on GPs' time and resources.

An interesting area for future research would be to find out more about cognitive and affective constructs surrounding 'hypothetical others', and what part the media play in informing these constructs.

This study on adults reporting cancer 'alarm' symptoms highlighted that the worry over wasting GP time was influenced by cognitive aspects (symptom characteristics and financial contributions to the NHS, GP responsibilities, in-appointment conduct) and emotive aspects (needs of hypothetical others, relationship with GP). Research is needed on how these attitudinal and emotive factors could be addressed in public health campaigns aimed at reducing time to presentation and improving earlier diagnosis of cancer. 


\section{REFERENCES}

1. Berrino F, De Angelis R, Sant M, et al. Survival for eight major cancers and all cancers combined for European adults diagnosed in 1995-99: results of the EUROCARE-4 study. Lancet Oncol 2007; 8(9): 773-783.

2. Butler J, Foot C, Bomb M, et al. The international Cancer Benchmarking Partnership: an international collaboration to inform cancer policy in Australia, Canada, Denmark, Norway, Sweden and the United Kingdom. Health Policy 2013; 112(1-2): 148-155

3. Renzi C, Whitaker KL, Winstanley K, et al. Unintended consequences of an 'all-clear' diagnosis for potential cancer symptoms: a nested qualitative interview study with primary care patients. Br J Gen Pract 2015; DOI: 10.3399/ bjgp16X683845.

4. Andersen RS, Vedsted P, Olesen F, Søndergaard J. Does the organizational structure of health care systems influence care-seeking decisions? A qualitative analysis of Danish cancer patients' reflections on care-seeking. Scand J Prim Health Care 2011; 29(3): 144-149.

5. Whitaker KL, Smith CF, Winstanley K, Wardle J. What prompts help-seeking for cancer 'alarm' symptoms? A primary care based survey. Br J Cancer 2016; 114(3): 334-339.

6. Whitaker KL, Scott SE, Winstanley K, et al. Attributions of cancer 'alarm symptoms in a community sample. PloS One 2014; 9(12): e114028.

7. Brindle L, Pope C, Corner J, et al. Eliciting symptoms interpreted as normal by patients with early-stage lung cancer: could GP elicitation of normalised symptoms reduce delay in diagnosis? Cross-sectional interview study. BMJ Open 2012; 2(6): pii: e001977.

8. Macleod U, Mitchell ED, Burgess C, et al. Risk factors for delayed presentation and referral of symptomatic cancer: evidence for common cancers. $\mathrm{Br} J$ Cancer 2009; 101(suppl 2): S92-S101.

9. Vrinten $\mathrm{C}$, van Jaarsveld $\mathrm{CH}$, Waller J, et al. The structure and demographic correlates of cancer fear. BMC Cancer 2014; 14: 597.

10. Forbes LJ, Warburton F, Richards MA, Ramirez AJ. Risk factors for delay in symptomatic presentation: a survey of cancer patients. Br J Cancer 2014; 111(3): $581-588$

11. Whitaker KL, Macleod U, Winstanley K, et al. Help seeking for cancer 'alarm symptoms: a qualitative interview study of primary care patients in the UK. $\mathrm{Br} J$ Gen Pract 2015; DOI: 10.3399/bjgp15X683533.

12. Hvidberg L, Wulff CN. Pedersen AF, Vedsted P. Barriers to healthcare seeking beliefs about cancer and the role of socio-economic position. A Danish population-based study. Prev Med 2015; 71: 107-113.

13. Smith LK, Pope C, Botha JL. Patients' help-seeking experiences and delay in cancer presentation: a qualitative synthesis. Lancet 2005; 366(9488): 825-831.

14. Robb K, Stubbings S, Ramirez A, et al. Public awareness of cancer in Britain: a population-based survey of adults. Br J Cancer 2009; 101(suppl 2): S18-S23.

15. Whitaker KL, Cromme S, Winstanley K, et al. Emotional responses to the experience of cancer 'alarm' symptoms. Psychooncology 2016; 25(5): 567-573.

16. BBC. NHS to reveal cost of missed appointments to patients. http://www.bbc. co.uk/news/uk-33375976 (accessed 9 May 2016).

17. Scott S, Walter F. Studying help-seeking for symptoms: the challenges of methods and models. Soc Personal Psychol Compass 2010; 4(8): 531-547.

18. Walter F, Webster A, Scott S, Emery J. The Andersen Model of Total Patient Delay: a systematic review of its application in cancer diagnosis. J Health Serv Res Policy 2012; 17(2): 110-118.

19. NHS Choices. Improving outcomes: a strategy for cancer. 2011. https://wnw. gov.uk/government/uploads/system/uploads/attachment_data/file/213785/ dh 123394.pdf laccessed 9 May 2016).

20. Independent Cancer Taskforce. Achieving world-class cancer outcomes: a strategy for England 2015-2020. http://www.cancerresearchuk.org/sites/ default/files/achieving_world-class_cancer_outcomes_-_a_strategy_for_ england_2015-2020.pdf (accessed 9 May 2016).

21. Cancer Research UK. Cancer incidence by age. http://www.cancerresearchuk org/health-professional/cancer-statistics/incidence/age\#heading-Zero laccessed 9 May 2016).

22. Low EL, Whitaker KL, Simon AE, Sekhon M, et al. Women's interpretation of and responses to potential gynaecological cancer symptoms: a qualitative interview study. BMJ Open 2015; 5(7): e008082.

23. McLachlan S, Mansell G, Sanders T, et al. Symptom perceptions and helpseeking behaviour prior to lung and colorectal cancer diagnoses: a qualitative study. Fam Pract 2015; 32(5): 568-577. 